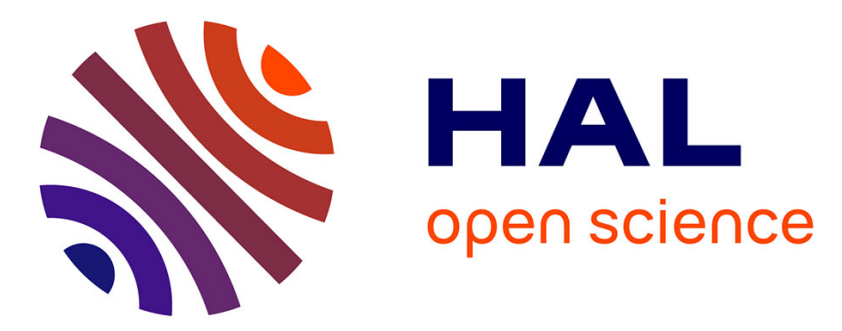

\title{
Expression de l'appartenance et de la possession dans le syntagme nominal en sudarabique moderne
}

\author{
Marie-Claude Simeone-Senelle
}

\section{To cite this version:}

Marie-Claude Simeone-Senelle. Expression de l'appartenance et de la possession dans le syntagme nominal en sudarabique moderne. Alessandro Bausi, Alessandro Gori and Gianfrancesco Lusini. Linguistic, Oriental and Ethiopian Studies in Memory of Paolo Marrassini, Harrassowitz, pp.661-687, 2014, 978-3-447-10317-6. halshs-01111714

\section{HAL Id: halshs-01111714 https://shs.hal.science/halshs-01111714}

Submitted on 30 Jan 2015

HAL is a multi-disciplinary open access archive for the deposit and dissemination of scientific research documents, whether they are published or not. The documents may come from teaching and research institutions in France or abroad, or from public or private research centers.
L'archive ouverte pluridisciplinaire HAL, est destinée au dépôt et à la diffusion de documents scientifiques de niveau recherche, publiés ou non, émanant des établissements d'enseignement et de recherche français ou étrangers, des laboratoires publics ou privés. 


\title{
Expression de l'appartenance et de la possession
}

\section{dans le syntagme nominal en sudarabique moderne}

\author{
Marie-Claude Simeone-Senelle. CNRS-France
}

\begin{abstract}
Avant-propos
Cette contribution se propose de présenter, à partir de l'étude des langues sudarabiques modernes, les moyens linguistiques permettant d'exprimer une relation d'appartenance ou de possession établie entre deux noms ou entre un nom et un substitut pronominal. En examinant un aspect particulier du système nominal, dans un groupe de langues sémitiques méridionales, parlées dans la péninsule arabique, l'article a pour but de participer à une meilleure connaissance de la morpho-syntaxe nominale en sémitique. Il répond aussi, malheureusement trop tardivement, à une sollicitation du professeur Paolo Marrassini, et il est une manifestation de l'estime portée aux recherches de ce grand sémitisant.
\end{abstract}

\section{Introduction}

Après un bref aperçu sur les langues concernées, j'aborderai la description des deux types de construction qui permettent d'exprimer en sudarabique moderne la relation d'appartenance et la possession : la construction directe, où seul l'ordre des constituants juxtaposés est pertinent, et la construction indirecte, où une marque spécifique lie les deux constituants de l'annexion. Quand les deux constructions coexistent dans une même langue, les valeurs sémantiques qui sont rattachées à chacune seront abordées. On dégagera à la fois les points communs et les divergences à l'intérieur du groupe, les traits atypiques qui singularisent une langue par rapport aux autres, afin de permettre d'éclairer d'un point de vue typologique et comparatiste les rapports à l'intérieur du sémitique méridional entre sudarabique moderne et des langues afro/éthio-sémitique. ${ }^{1}$

\section{Les données}

Pour les langues parlées au Yémen, les données reposent essentiellement sur le corpus que j'ai relevé sur le terrain, lors de missions menées régulièrement entre 1983 et fin 2010, au Yémen. Cela concerne le mehri et ses deux principaux dialectes (mehriyet et mehriyot), le hobyot et le soqotri. ${ }^{2}$ Pour les langues parlées en Oman - bathari, harsusi, jibbali et mehri d'Oman -elles sont empruntées aux sources éditées. Dans ce cas, la référence est toujours notifiée à côté de l'exemple.

\footnotetext{
${ }^{1}$ Un travail en cours met en parallèle ces constructions avec celles relevées en dahalik, tigré et tigrinya.

2 Les missions ont été financées par le CNRS, le Ministère français des Affaires étrangères, le Centre français (CFEY, désormais CEFAS), à Sanaa. Elles ont aussi bénéficié, au Yémen, du soutien de l'Université d'Aden.
} 


\section{Remerciements}

Je tiens à exprimer ma profonde gratitude et ma reconnaissance à tous les locutrices et locuteurs de langue mehri, hobyot, et soqotri qui ont collaboré activement à ces recherches en me faisant partager leur connaissance.

\section{Conventions de lecture et abréviations}

La transcription des auteurs a été, dans la mesure du possible, respectée.

Aux symboles utilisés pour l'arabe et ses dialectes, sont ajoutés ceux qui permettent de rendre compte du système consonantique propre à ces langues : ś pour la latérale fricative sourde (API : \), avec une variante sonorisée notée ź (API 3), c'est aussi l'allophone fricatif de /1/ (Johnstone 1981: xiv); la latérale

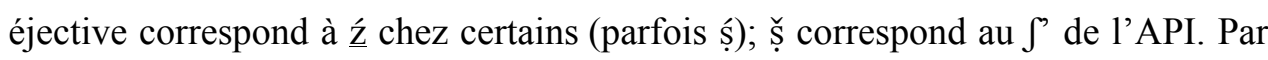
convention, les consonnes éjectives sont notées comme les emphatiques de l'arabe, avec un point souscrit. La notation des interdentales a été harmonisée : $\neq$, pour $\theta$ ou t selon les auteurs, et đ, pour $\partial$ et $\underline{\mathrm{d}}$.

J'ai rajouté le mot-à-mot ou les gloses manquants dans les exemples issus d'autres sources que mon corpus.

ACC. accompli - AUX. auxiliaire $-\mathrm{D}^{\text {ant }}$ déterminant $-\mathrm{D}^{\mathrm{e}}$ déterminé - DEF article défini - DEIC déictique - DEM démonstratif - DIST distal - F féminin - EP épenthèse - GEN génitif - IN indice de nombre - INAC inaccompli - INT interrogatif - M masculin - NEG négation - PERS personnel - PL pluriel - POS possessif - PR pronom - PREP préposition - PROX proximal - REL relateur - SG singulier - SN syntagme nominal - SUF suffixe/é - v voyelle- = est la marque de clitique.

\section{Autres abréviations :}

Nom des langues

B. batḥāri - J jibbāli ${ }^{3}-\mathrm{Hb}$ hōbyōt - Ḥ hạsūs̄ī - M mehri (sans distinction dialectale) - Met mehriyet (parler au Yémen, région sud-ouest du Mahra) - MO mehri parlé en Oman (relevé dans ML et Str.) - Mot mehriyot (parler au Yémen, est du Mahra) - S soqotri.

Quand les données ne proviennent pas du corpus de l'auteur, elles sont référenciées comme suit :

ḤL Harsūsi Lexicon (Johnstone 1977)- JL Jibbāli Lexicon (Johnstone 1981) - LS Lexique șoqoțri (Leslau 1938) - ML Mehri Lexicon (Johnstone 1987) - M (Str) Mehri Texts (Stroomer 1999) - Ḥ (Str.) Harsūsi Texts (Stroomer 2004) - M (Sima) Mehri-Texte (Sima 2009).

Les exemples extraits des textes publiés comportent le numéro de la page suivi du numéro du $\S$.

\footnotetext{
${ }^{3}$ jibbāli, ce nom, donné à la langue par Johnstone, est actuellement contesté par beaucoup de ses locuteurs qui la nomme śḥcri ('(celle) de la montagne').
} 


\section{Les langues}

Les langues sémitiques méridionales parlées dans le sud de la péninsule Arabique sont regroupées sous le terme collectif de sudarabique moderne (= SAM). Elles constituent un ensemble bien délimité géographiquement et linguistiquement ${ }^{4}$. Ce sont des langues maternelles dans deux états qui ont l'arabe pour unique langue officielle: au Yémen, dans la province orientale du Mahra et sur trois îles de l'archipel de Soqotra, et en Oman, à l'ouest du sultanat. Elles sont au nombre de six : mehri, ${ }^{5}$ hobyot, harsusi, bathari, soqotri et jibbali ${ }^{6}$. Les quatre premières sont très proches typologiquement, alors que le soqotri d'une part et le jibbali d'autre part présentent des caractéristiques qui leur sont propres.

\section{Typologie}

Les langues SAM sont des langues dont l'ordre canonique est VSO dans la phrase verbale; Aux. $+\mathrm{V}$, dans le syntagme verbal.

Dans le syntagme nominal : N + ADJ, N.D ${ }^{\mathrm{e}}+\mathrm{N} \cdot \mathrm{D}^{\text {ant }} ; \mathrm{N}+$ DEIC.

Seules les langues parlées en Oman ont un article défini (invariable), toujours préclitique au nom défini.

\section{Les constructions génitivales et la possession}

La relation de possession et d'appartenance, instaurée entre deux éléments (deux noms ou un nom et un substitut nominal), est exprimée par des constructions souvent nommées "génitivales". Un des deux constituants du syntagme est dépendant de l'autre, ${ }^{7}$ il en est le complément, il a une fonction de déterminant, et selon le sémantisme des deux constituants, il réfère à celui "à qui appartient" ( $c f$. (1)) ou "celui / ce qui appartient à" ( $c f$. (6)). Le nom, dans les langues concernées, n'est pas soumis à la flexion casuelle, cette relation est donc marquée syntaxiquement, soit par une construction directe, dite aussi synthétique (ou état construit), dans laquelle les deux composants sont juxtaposés, soit par une construction indirecte, dite aussi analytique. Dans ce cas, la marque de construction est explicitée par un morphème. Il peut être spécifique ou assumer une fonction de joncteur, relateur, connectif, entre les constituants d'un syntagme nominal. On le glosera ici par GEN. (pour marque de génitif).

Les deux constructions peuvent coexister dans une même langue, mais elles ne sont pas toujours équivalentes sur le plan sémantique. L'emploi de l'une ou de l'autre dépend à la fois de la valeur sémantique de la relation instaurée entre les deux constituants et, dans certaines de ces langues, de la catégorie grammaticale à laquelle appartient le déterminant (nom ou pronom). L'ordre des deux constituants est le même dans toutes les langues, $\mathrm{D}^{\mathrm{e}}+\mathrm{D}^{\text {ant }}$, excepté en soqotri,

\footnotetext{
${ }^{4}$ Cf. Simeone-Senelle 2011.

5 Le mehri comprend trois groupes dialectaux principaux : məhrīyet et mehriyōt (au Yémen), məhráyyət (en Oman).

${ }^{6}$ hōbyōt / həwbyot ; ḥarsūsi ; bațhāri ; jibbāli ; soqoṭri [sḳ́ț̣i].

${ }^{7}$ Les sémitisants distinguent traditionnellement dans la construction génitivale le 'nomen rectum' et le 'nomen regens' (Lutz 2009).
} 
quand le référent du possesseur est un pronom personnel. Dans le cas des constructions complexes, l'élément déterminant est constitué par un syntagme nominal génitival, composé de deux noms ou d'un nom et d'un déterminant possessif.

Il sera d'abord question de la construction directe entre deux noms ou entre un nom et un référent pronominal, puis de la construction indirecte entre deux noms ; les constructions complexes seront abordées en fin d'article.

\section{1. Construction directe}

Cette construction est la moins fréquente, elle ne concerne que des noms appartenant à des champs sémantiques restreints. Le nom référent du possédé appartient à la sphère personnelle du possesseur. ${ }^{8}$ L'état construit permet d'énoncer une relation de possession inaliénable entre $\mathrm{D}^{\mathrm{e}}$ et $\mathrm{D}^{\text {ant }}$. On la trouve essentiellement avec un nom qui désigne une partie intrinsèque au possesseur (noms de parties du corps, de parenté), ou dans les toponymes et les noms de tribus ou de clans où la relation établie est celle d'une appartenance définitoire.

$$
\text { II. 1.1. } N+N
$$

L'ordre est immuable : le déterminé est le premier constituant du syntagme, le déterminant lui est apposé. La seule marque de fonction réside dans l'ordre des termes.

La construction est attestée dans les six langues mais elle reste très limitée et a tendance à être remplacée par la construction analytique ( $c f$. ci-dessous). Déjà Bittner (1913 : 64), à partir de l'étude de textes mehri édités au tout début du $20^{\text {ème }}$ siècle, remarquait que la construction directe n'apparaissait pas comme très fréquente. ${ }^{9}$

Dans les corpus contemporains consultés, comme dans mes données, les occurrences les plus nombreuses de la construction marquent la filiation, l'appartenance à une communauté, à une région, à un statut social (lié à la généalogie). Le $\mathrm{D}^{\text {ant }}$ est souvent un nom propre.
(1) B ber faliy (Morris 136)
fils Ali
'Son of Ali'
(2) M bərt-sālem [bəttsālem] (Met \& Mot)
fille-salem
'Fille de Salem'

(3) $\mathrm{Hb}$ seh bərt-ənsīb

PR.3F.SG fille-Nasîb

\footnotetext{
${ }^{8}$ Cf. Creissels 2006: 57, à propos des noms avec affixes possessifs 'qui signifient le rattachement à la sphère personnelle d'un référent saillant (c'est-à-dire d'un référent qui serait susceptible, dans d'autres contextes syntaxiques, d'être représenté par un pronom personnel)'

9 'Nicht gerade allzu häufig'.
} 
'Elle (est) (la) fille de Nasîb'

(4) S yíhin bər-șațhan (parler de la côte nord)

PR.3M.SG fils-sultan

'Lui, (il est) le fils du sultan'

Avec les noms de clans ou de tribus :

(5) Met habenti-āf ${ }^{\ominus} r \bar{r} \quad$ bìt = āf`rīr

filles-' Afrir tribu='Afrir

'Filles des 'Afrir, les 'Afrir'

La construction du type Bit'Afrïr est figée, comme dans tous les noms de tribus, et peut fonctionner comme un nom propre. bīt=solōm 'les Bit Solom', bīt=bakāret 'les Bit Baqaret' ...

La construction directe est très courante après le nom désignant 'maître, responsable de, expert en, propriétaire, celui/celle qui détient' : bål ${ }^{10}$ (et ses variantes selon les langues) qui varie en genre et dont le pluriel (commun) a le sens de 'gens, peuple'. Elle permet de marquer qu'il existe un lien très étroit d'appartenance et de possession entre les deux constituants ( $c f$. plus bas la différence sémantique avec la construction indirecte), le $\mathrm{D}^{\text {ant }}$ dans ce cas réfère à ce qui est possédé en propre par le $\mathrm{D}^{\mathrm{e}}$.

(6) Met bālet hārūn (parler bédouin)

maîtresse- chèvres

'Bergère/ chevrière' (elle est propriétaire des chèvres et non simplement leur gardienne)

(7) $\mathrm{S}$ baial béier (parler de la côte orientale)

maître camélidés

'Chamelier' (à qui le troupeau appartient).

Avec le pluriel, le syntagme est figé en un nom composé ayant son sens propre. Ainsi dans la formule de salutation utilisée en mehri, pour demander des nouvelles de toute la famille :
(8) $\mathrm{M} \quad$ bōli=béyt
b-xeyr
(Mot \& Met)
gens_de_la_maison
PREP-bien
'La famille (la maisonnée) se porte bien ?'

Le singulier, mais surtout le pluriel, suivi d'un toponyme permet de désigner les habitants d'une région, d'un village. C'est leur appartenance à ce lieu qui les définit.

(9)

Met bōli-ḳấśn ' les habitants/ les gens de Qishn’

${ }^{10}$ La racine $<$ b $>$ est attestée et recouvre le même champ sémantique en sémitique et dans beaucoup de langues couchitiques. 
Met bōli-msílet 'les gens du (Wadi) Masila'

Met bōli-ấmēn 'les gens de 'Oman, les Omanais'

Mot bạli-hú "byōt 'les Hobyot, la communauté hobyot'

B bəfēli śerbițōt 'the people of Sharbithhat' (Morris 1983 : 134)

Le composé peut aussi fournir une nouvelle entrée lexicale. C'est le cas des constructions où le déterminé est ber-, au sens propre 'fils de, descendant'. En (10) bər n'est pas complètement vidé de son sens de nom de parenté :

(10)S bər = ḳākāā

enfant- frère/sœur

'Neveu/nièce'

ni dans l'exemple suivant, tiré d'un texte littéraire ${ }^{11}$ (Müller 1905, 18, 7). C'est par le biais de cette construction (et le recours au lexique arabe) que le locuteur a forgé un nouveau nom pour désigner un petit d'animal non référencié en soqotri : ,ber-Pésed 'lionceau' ('ein jünger Löwe').

Par contre, dans certains noms composés, ce même terme a un champ sémantique qui ne se restreint pas au lien de parenté génétique et il prend le sens de 'sorte de/ dérivé de'. Le déterminant pouvant être un N (+/- animé). Des noms d'animaux, de plantes, de phénomènes météorologiques sont ainsi formés. bər= est en tête de syntagme et proclitique du nom (ou du SN) qui suit.

En (11), la construction devient un nom de vent.

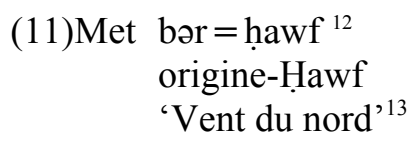

Quand le syntagme a un degré de lexicalisation très élevé, il peut être impossible d'en retrouver les composants.

En Met, bərkendūfiḳay désigne des échassiers (héron, aigrette, flamant...).

On peut décomposer le nom en bər=kendu=fikay $<\mathrm{fk} \varsigma>$. On y reconnaît bər, et fiḳay qui réfère à 'petite étendue/ trou d'eau, retenue d'eau de pluie', lieu fréquenté par ces oiseaux. Le deuxième élément jusqu'à maintenant reste indéchiffrable.

L'exemple en H (Str. 24, 32) : mehri bāl karmáyn est traduit par 'the Mehris, the lords of the mountain' (Str. 25, 32). Il s'agit d'une métonymie, comme le montre l'emploi des deux singuliers (mehri et bāl) et la traduction littérale est 'Le Mehri, seigneur de la montagne'.

La construction directe est aussi attestée dans certains toponymes :

(12) $\mathrm{Hb}$ bunti = márbōṭ $($ en Mot mənti = et en Met bənti =)

\footnotetext{
${ }^{11}$ Traduction d'un passage de la Bible en soqotri.

12 Relevé aussi par Jahn $(1902,134,3$; 169), repris par Wagner $(1953,73)$ que cite Watson (2009: 232).

${ }^{13}$ Ḥawf est un village à la frontière entre le Mahra et Oman, au nord-est de la région où se parle le Met.
} 
région-Marbôt

'(la région de) Marbôt'

Le syntagme ainsi formé constitue une seule unité accentuelle. Il apparaît comme sémantiquement figé, équivalent à un nom propre.

Le déterminant est définitoire, il permet de spécifier le déterminé par un trait qui lui est exclusif. D'autres exemples illustrent cette valeur :

(13) Mot qāro?-ğâdeb = Met ġəroy ğốdəb

langue-Jâdib

'Le parler de Jâdib'

En soqotri, la construction analytique n'a été relevée qu'avec bor- référant à un nom de parenté.

Les langues parlées en $\operatorname{Oman}^{14}$ marquent la définitude par un article invariable pré-clitique du nom. ${ }^{15}$ Dans les constructions génitivales directes, selon le contexte : il peut n'y avoir aucun élément défini, un des constituants seulement ou les deux. Les possibilités sont :

$$
\begin{aligned}
& (\mathrm{DEF}=) \mathrm{N} \cdot \mathrm{D}^{\mathrm{e}}+(\mathrm{DEF}=) \mathrm{N} \cdot \mathrm{D}^{\text {ant }} \\
& \text { ou } \mathrm{DEF}=\mathrm{N} \cdot \mathrm{D}^{\mathrm{e}}+\mathrm{N} \cdot \mathrm{D}^{\text {ant }} \text { ou N.D } \mathrm{D}^{\mathrm{e}}+\mathrm{DEF}=\mathrm{N} \cdot \mathrm{D}^{\text {ant }} .
\end{aligned}
$$ $\begin{array}{lll}\text { (14) M } & \begin{array}{l}\text { befēli } \\ \text { gens/propriétaires }\end{array} & \text { abdén } \\ \text { DEF.bateau } & 16\end{array} \quad$ (Str. 218, 2 \& 220, 5)
'The owners of the beden'

La construction avec le $2^{\text {ème }}$ élément défini peut aussi se figer et constituer une entrée lexicale :
(15) $\mathrm{H}$
bāl aṣayd (équivalent de bāl ḥẹt )
responsable DEF.poisson
'Fisherman' (lit. en charge du poisson)

Le fait, que le seul pluriel attesté soit hewwōt (ḤL 117), montre que la construction ne s'est figée lexicalement qu'au singulier.
(16) MO howrēd
amener_au_point_d'eau.ACC.3MPL gens DEF.caprins

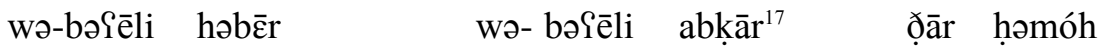
et-gens DEF.camélidés et-gens (DEF)bovins sur eau
'The goatherders, camel-herders and cowherds drove (their beasts) down to the water' (ML 41).

\footnotetext{
${ }^{14}$ C'est là un trait distinctif, assimilé à une isoglosse, entre les langues SAM parlées au Yémen (mehri, hobyot et soqotri) et celles parlées en Oman (mehri, jibbali/sheri, harsusi, bathari).

${ }^{15}$ L'article défini n'apparaît pas devant tous les noms, sa présence est conditionnée par la qualité du phonème initial du nom défini. Il est la plupart du temps une voyelle (HL xiv, JL xxix, ML 1).

16 bədén : 'kind of boat' (ML, 43).

17 abḳār est la forme du pl. définie ou non.
} 
Il faut remarquer que dans les constructions de ce type, quand le N.D $\mathrm{D}^{\text {ant }}$ est défini par un article qui est une voyelle (pré-clitique au nom) et qu'il suit un N.D ${ }^{\mathrm{e}}$ au singulier, le défini peut être confondu formellement avec le morphème marqueur de génitif.

Seul le sémantisme de la relation peut permettre de désambiguïser la construction (directe ou indirecte ?). En B. dans les textes relevés (Morris 1983), seul se construit directement le nom de parenté ber 'fils', l'exemple (17) ne peut donc se confondre avec une construction directe où le $2^{\text {eme }}$ constituant est défini, de plus elle n'instaure pas une relation d'appartenance ou de possession inaliénable : le $\mathrm{e}=$ clitique de xaarbut est vraisemblablement le marqueur de la construction génitivale indirecte ( $c f$. aussi (55)).

$$
\begin{aligned}
& \text { befaal exaarkút } \\
& \text { propriétaire GEN/DEF.blague_à tabac } \\
& \text { 'The owner of the tobacco pouch' }
\end{aligned}
$$

(Morris 139, 23)

Par contre l'ambiguïté peut subsister dans (18 \& 19)

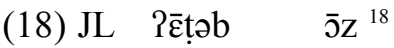

$$
\begin{aligned}
& \text { DEF.pis GEN/DEF.chèvre } \\
& \text { 'The teat of goat' } \\
& \text { (19) JL baial Érún }{ }^{19} \\
& \text { responsable GEN.DEF.chèvres } \\
& \text { 'Goatherd' }
\end{aligned}
$$

\section{1.2. $N=$ PR.PERS.DEP.}

Quelle que soit la nature de la relation instaurée entre les deux constituants de la construction (possession alénable ou non), lorsque le déterminant est un substitut pronominal, il réfère au 'possesseur', il a la forme du pronom personnel dépendant, ${ }^{20}$ et il est post-clitique au déterminé. On est dans le cadre d'une construction directe dont l'ordre est toujours $\mathrm{D}^{\mathrm{e}}=\mathrm{D}^{\text {ant }}$. Il n'y a pas distinction syntaxique entre une relation d'appartenance inhérente ou non. Le soqotri constitue une exception notable en SAM (cf. II.2.2.), il faut aussi prendre en compte, dans les autres langues, de très rares constructions indirectes impliquant une modalité particulière ( $c f$. ci-dessous : Remarque).

$$
\begin{array}{lll}
\text { (20) M } & \begin{array}{l}
\text { beyt }=\mathrm{i} \\
\text { maison=SUF.1SG }
\end{array} & \text { 'ma maison' } \\
\text { Met } & \begin{array}{l}
\text { biśrrt }=\mathrm{iš} \\
\text { cadeau=SUF.2FSG }
\end{array} & \text { 'ton cadeau' }
\end{array}
$$

\footnotetext{
${ }^{18}$ La forme définie de (?)oz est (?)̄̄z (JL5). La voyelle /e/ s'assimile à 0 . La marque de GEN ( $\varepsilon$ ou e) peut aussi s'assimiler à l'initiale du mot régi.

${ }^{19}$ L'indéfini étant crún (JL 4), la présence du GEN ou du DEF entraîne l'allongement de la voyelle (ērún) ou son accentuation (érún).

${ }^{20}$ Qui peut avoir une forme particulière selon qu'il est clitique d'un nom, d'un verbe ou d'une préposition (Simeone-Senelle $2011: 1083$ ).
} 
Mot $\quad$ Saydähäm

sardines.SUF.3MPL 'ihre Sardinen’ (Sima 268, 12)

$\mathrm{Hb}$ lheti = yin 'nos vaches'

vaches $=$ SUF.1PL

Dans les langues qui ont un article, le $\mathrm{D}^{\mathrm{e}}$ est très souvent défini. Seule la forme définie du nom de parenté, désignant 'le fils, la fille, les enfants, parfois la mère et le père', peut avoir un suffixe référant au possesseur. Dans les langues sans article c'est une autre forme (de même racine) à une forme figée qui est employée ${ }^{21}$.

En soqotri, on remarque que le déterminant possessif ne peut se suffixer qu'à un petit nombre de noms de parenté et ces noms ont une forme différente quand ils sont en état d'annexion avec un autre nom ou quand le pronom personnel est régi par la marque GEN. (et tout le syntagme possessif préposé au nom déterminé). ${ }^{22}$

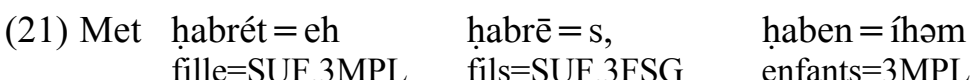

'Sa fille, son fils, leurs enfants'

(22) MO hạāmēh (Str. 30,9)

mère.SUF.3MSG : 'his mother'

(DEF) amendáwḳeh (ML 2) $(/ \mathrm{a}=$ mendáwk = eh)

DEF.fusil.SUF.3MSG : 'his rifle'

(23) B šebdéti (Morris 1983 : 132, 2)

foie.SUF.1SG : 'my liver'

(DEF) efayniy (Morris id.) (/e = Iayn = iy/)

DEF.œil.SUF.1SG : 'my eye'

(24) H frékeh (Str. 38, 25)

campement. 3SUF.MSG : 'his community'

(DEF) ag̉aygeš $($ Str. 49,37$)(/ \mathrm{a}=$ gayg $=$ eš $/)$

DEF.homme.SUF.2FSG : 'your husband'

(25) JL totš (JL 300)

femme.SUF.3MSG : 'his wife'

(DEF) edídésən $(J L 42)(/ e=$ díd $\varepsilon=$ sən/)

DEF.oncles.SUF.3FPL : 'their (f.) uncles'

En jibbali, la construction sans DEF est rare. ${ }^{23}$

21 Les lexèmes sont figés avec ha-/hạ- (selon les parlers) comme préformantes. Ils prennent souvent un sens défini. Certains voient dans ces formes une trace de l'article défini.

${ }^{22}$ En S., la construction directe avec pronom suffixé n'est possible qu'avec $\mathrm{ibr}=\mathrm{hi}$ 'mon enfant' ou au f. Gibrit- ( $c f$.LS 95), byoh 'mère' ne s'emploie jamais avec un pronom suffixe (LS 81) pas plus que bébeh 'père', seul le nom supplétif Giif supporte la construction directe avec pronom post-clitique (LS 80).

${ }^{23}$ Sur 23 exemples relevés dans HL avec un pronom 3MSG : 13 comportent le DEF. En 
En $H$, cette construction est fréquente avec mōl $<\sqrt{ }$ mwl $>$ qui désigne les biens essentiellement constitués par le bétail, $c f$. (HL 92) 'flocks, livestock, property'. Dans HL, tous les exemples sont traduits par 'folks' dans HL.

(26) $\mathrm{H}$ amōleh- $(/ \mathrm{a}=\mathrm{mōl}=\mathrm{eh} /)^{-}$-hah DEF.biens.SUF.3MSG- -PR.3MSG 'His flocks'

$$
\begin{array}{ll}
\text { amōlsen } / \mathrm{a}=\mathrm{mō} l=\mathrm{sen} / \text { ) } & -\mathrm{sē}^{24} \\
\text { DEF.biens.SUF.3FPL- } & -\mathrm{PR} .3 \mathrm{FPL} \\
\text { 'Their(f.) flocks' } &
\end{array}
$$

La relation d'appartenance peut être renforcée par la postposition au syntagme d'un pronom personnel indépendant, anaphorique, référant au possesseur $/ \mathrm{D}^{\text {ant. }}$. Cette construction est surtout fréquente quand le pronom renvoie au locuteur.
(28) Met beyt $=\mathrm{i} \quad$ hoh maison= SUF1SG PR.1SG
'Ma maison à moi, ma propre maison'
(29) $\mathrm{H}$ feréki hoh (Str. 38, 5)
campement.SUF.1SG PR.1SG
'My encampement'
(30) B Paatər'tiy ho (Morris $1985: 140,31$ ) milk skin.SUF.1SG PR.1SG 'My milk skin'

Huit exemples de ce type ont été relevés dans HLL.

Quand le déterminant réfère à un pluriel ou un duel (32), il est explicité par un pronom indépendant toujours juxtaposé au SN et un nom.
(31) H hẹi hoh (we-háwbikem) (Str. 8, 17) père.SUF.1SG PR.PERS.1SG (et-DEF.pères.SUF.2PL) 'My father' (= mon propre père, mon père) (and your fathers)
(32) H amōlki ${ }^{25} \quad$ het w-areybyāk DEF.biens.SUF.2DU PR..PERS.2MSG et-

'The flock of you and your companions'(Lit. 'vos troupeaux à vous deux: toi et le groupe de tes compagnons)

REMARQUE SUR LA CONSTRUCTION INDIRECTE AVEC PRONOM PERSONNEL INDEPENDANT

JL, sur 56 exemples, seuls 11 ne sont pas définis par l'article . Quant aux données de B, elles sont trop peu nombreuses pour émettre une estimation.

${ }^{24} \mathrm{La}$ forme du pronom dépendent pour la 3F.PL (=sen), diffère bien de celle du pronom indépendant sēn.

$25 / \mathrm{a}=\mathrm{mō}=\mathrm{ki} /$. 
En soqotri, c'est la construction qui domine quand le référent au possesseur est un pronom. Dans les autres langues SAM, le syntagme composé du morphème $\mathrm{d} / \mathrm{d}+\mathrm{PR}$. PERS indépendant a une fonction de pronom possessif $^{26}$, il est toujours postposé au $\mathrm{N}$ référant au possédé, l'ordre de la construction directe est maintenu. Les exemples relevés, uniquement en Met, $\mathrm{Hb}$ (du Yémen) et en MO, sont très rares. En Met (33), la construction est postposée au syntagme N+PR.SUF, en $\mathrm{Hb}$ (34)et MO (35), il est juste postposé au nom. La marque de GEN est aussi un REL/CON. ${ }^{27} \mathrm{La}$ construction dans ces exemples est celle d'une relative nominale, appositive en Met, et déterminative en $\mathrm{Hb}$ et MO. La possession est explicitée par une phrase nominale, ce renforcement de la détermination a une valeur modale d'insistance, de revendication quant à l'identification du possesseur.

Avec reprise du pronom dépendant :

$$
\begin{array}{cll}
\text { Met } & \text { sakker }=\mathrm{k} & \mathrm{d}=\mathrm{h} \overline{\mathrm{t}} \\
& \text { sucre=PR.SUF.2M.SG } & \text { REL=PR.2SG } \\
& \text { 'Ton sucre, le tien (qui est à toi)' }
\end{array}
$$

Sans reprise

(34) $\mathrm{Hb}$

$$
\begin{array}{ll}
\text { lhêtə } & \text { đə=ti } \\
\text { vaches } & \text { REL=PR.1/2DU. }
\end{array}
$$

Nos vaches (à nous deux)' (qui (sont à) nous deux) ou 'Les vaches, les nôtres'

$$
\begin{array}{cllll}
\text { (35) MO } & \text { đōmeh } & \text { adabh } & \text { đe-ho } & \text { (Str. 208, 7) } \\
& \text { DEM.M.SG } & \text { DEF.miel } & \text { REL-PR.1SG } & \\
\text { 'This is my honey' ou 'c'est le miel qui m'appartient' }
\end{array}
$$

Ces constructions sont liées à celles des relatives, elles permettent d'exprimer la possession mais n'entrent pas dans une construction génitivale.

\section{2. La construction génitivale indirecte.}

Toutes les langues SAM acceptent une construction directe et une construction indirecte quand les deux constituants du syntagme en rapport d'annexion sont deux noms. Des critères sémantiques induisent une construction plutôt qu'une autre. Cependant la construction indirecte domine car elle n'est pas restreinte lexicalement et elle peut avoir la même valeur que la construction directe. Nous avons vu que si le possesseur a un pronom personnel comme référent, la construction directe, attestée dans toutes les langues ( $c f$. ci-dessus), en est la règle sauf en soqotri. Dans cette langue, la construction directe avec un pronom suffixé est rarissime, elle est cantonnée à quelques lexèmes appartenant à un champ sémantique restreint, et c'est une construction indirecte spécifique qui domine. Dans les six langues, le lien entre les deux constituants nominaux du syntagme est

${ }^{26} C f$. MO (Str. 200, 21) đōmeh đe-hō 'it is mine !' et (Str. 176, 8) hē đe-ho 'It is mine'.

${ }^{27}$ Cf. Simeone-Senelle 2003. 
marqué par un morphème (ou une particule) préfixé/enclitique au nom déterminant. Selon les auteurs, cette marque est glosée comme GEN (marque de génitif), marque de construction (génitivale), connectif, joncteur ou encore relateur. Nous avons ici choisi GEN.

\section{2.1. $N+G E N=N$}

La relation de dépendance entre les deux constituants est marquée morphologiquement, par un morphème variable en nombre, et syntaxiquement par l'ordre des termes qui est le même que dans les constructions directes.

Dans la majorité des langues SAM, le marqueur a la même base lexicale (au singulier et au pluriel) que celle du déictique masculin de proximité ou du relateur. ${ }^{28} \mathrm{Il}$ ne varie pas en genre (quel que soit le genre du déterminé et celui du déterminant), mais il peut varier en nombre lorsque le déterminé est un pluriel. C'est le nombre du déterminé qui induit celui de la marque de fonction. Cependant, comme nous le verrons dans les exemples ci-dessous, il y a de nombreuses entorses à cette règle d'accord et l'emploi du pluriel est rare dans certaines langues. Il y a une nette tendance à uniformiser la construction avec la marque sous sa forme singulier.

En M., Hb., H., et S., le marqueur est, au singulier : $\mathrm{d}(\mathrm{v})=(\mathrm{ou} d(\mathrm{v})=$ dans les langues ou dialectes sans interdentales). En B. et J. le morphème marquant une relation de possession est vocalique au singulier (e en B. et $\varepsilon$ en $J .{ }^{29}$ ). Si le déterminé est au pluriel, le morphème pré-clitique est $\mathrm{l}=$ dans toutes les langues (avec une réalisation iź= en J.). Sa forme correspond à la base pluriel du déictique, sauf en $\mathrm{B}$. où ce l- est une préposition ( $c f$. ci-dessous), qui marque le déterminant et peut suivre aussi bien un N. SG que PL.

Quand les deux constructions, directe et indirecte, coexistent, la première marque une possession inaliénable, alors que la seconde indique que le Dé et son Dant ne sont pas dans un rapport d'inhérence, qu'il s'agit d'une possession contractuelle, provisoire, de jure et non de facto.

(36) Met bālēt đə=hārūn (parler bédouin)

responsable $\mathrm{GEN}=$ caprins

'La bergère/la chevrière' (celle qui s'occupe des caprins)

Comparer avec (6) bālēt-hārū

De même en J :

$\begin{array}{ll}\bar{a} \text { @al (/DEF.baial/) } & \text { iź-írún } \\ \text { DEF.responsable } & \text { GEN.PL. = caprins } \\ \text { 'Goatherds' } & \end{array}$

'Goatherds'

Au singulier : āial ćrún 'goatherd'.

${ }^{28}$ Cf. Simeone-Senelle 2003, $2011: 1087$.

${ }^{29}$ En J. đ = marque une relation d'appartenance qui ne relève pas du rapport possédépossesseur ( $\mathrm{JL}: 44$ "đ is used where the relation is not one of possession"). 
En (37), la forme définie de baSal (ā̧al) suivi d'un GEN SG induit le SG du nom composé, suivi d'un GEN au PL. induit le PL. du nom. Le N. $\mathrm{D}^{\text {ant }}$ avec la marque de GEN. forme une seule unité, se fige et a un sens de PL: iź-írún (JL 4) 'goatherds', 'goat people'(JL 234), lit. 'ceux des caprins' (cf. ci-dessous $(64,65)$, l'évolution du soqotri)

Lorsque le $\mathrm{D}^{\mathrm{e}}$ est défini par l'article, même s'il s'agit d'un nom de parenté, la construction est indirecte
(38) JL ébré $\quad \overline{\text { désós }}$
DEF.fils GEN.SG.DEF.serpent
'The son of the snake' (exemple tiré d'un proverb)
(39) MO hebrìt đ-așayyōd
DEF.fille GEN.SG-DEF.pêcheur
(Str. 266, $6 \& 8$ )
"The daughter of the fisherman'

En mehri du Yémen (Met et Mot), pour les noms de parenté (fils, fille, enfants), seuls les lexèmes avec préformante : ḩəbrē (m.), ḩəbrìt (f.) habūn (pl.), ${ }^{30}$ peuvent se construire avec un GEN marqué (cf. ML 54). ${ }^{31}$
(40) Met habūn le $=$ salēm (parler bédouin)
enfants GEN.PL.Salem
'Les enfants de Salem'
(41) Met ḥəbrē đ đ=haybìt (parler bédouin)
petit/fils GEN.SG $=$ chamelle
'Chamelon'

Cette construction est plus fréquente que l'état construit et on peut aussi la trouver avec des noms de parties du corps
(42) $\mathrm{Hb}$ ḳerd đo= ,ali
voix $\quad$ GEN.SG $=$ Ali

'La voix de Ali'
(43) B śerót đ-mílík (Morris 1985, 123, 3)
breath GEN-angel
'The (sweet) breath of an angel'

Des toponymes sont ainsi formés à partir de métaphore avec un nom de partie du corps, le constituant marqué est le plus souvent un nom propre :
(44) Met hərūh də= śárwī
tête/cap GEN.SG. $=$ malheur.DU
'Le cap des deux malheurs' ('cap Sharwîn', en arabe sur les cartes). ce cap délimite la baie de Qishn à l'ouest.

\footnotetext{
${ }^{30}$ Alors que ber, bert sont toujours en état construit.

${ }^{31}$ Watson $(2009: 230)$ relève ce trait sans le commenter.
} 
(45) JL kérún iź-hịróto (JL xiii)

cornes GEN.PL.montagnes

'Horns of the mountains' (nom d'une région dans le Dhofar)

(46) S házereh di=maḳdérihən

creux $^{32}$ GEN.SG.Maqderihen

'Le col de Maqderihen'

Le syntagme peut se lexicaliser, le nom composé qui en résulte a un sens qui lui est propre. Ainsi le nom de la 'murène léopard', résulte de la même construction en MO et en J.

(47) MO ekayźer đe ráwnem (Morris 1983, 138)

DEF.léopard GEN. mer

(48) J kéźer $\quad$ eremnem $^{33} \quad$ (Morris 1983, 138)

léopard GEN.mer

'moray eel' (lit. '(le) léopard de mer')

Dans certains de ces noms composés, il n'y a pas de variation en nombre du marqueur de GEN.
(49) Met Sađ̣̂mīt đə=ḥamsīt (parler bédouin)
dos GEN.SG.tortue
'Carapace' (Lit. 'dos de tortue')
(50) Met Sayđ̣̆m đə= himostən (parler bédouin)
dos (pl.) GEN.SG.tortues
'Carapaces' (Lit. 'les dos de tortues')

Par contre, cette variation est attestée dans une autre construction figée du même type :
(51) Met kasirirūt
écorce
də $=$ hamsit
\& kaśyōr $1=$ hemésten
écorce GEN.SG.tortues
écorces GEN.PL $=$ tortues

'Carapace' \& 'Carapaces' (Lit. 'écorce/s de tortue/s')

Après un collectif, la marque GEN. peut être au SG ou au PL.
(52) Met haǧerīt ${ }^{34} \quad 1=$ ḳāśən $[\ldots]$ hağerīt $\mathrm{d} ə=$ məsloy dépendants GEN.PL.Qishn dépendants GEN.SG= région de Masila
'Les Noirs de Qishn, [...] les Noirs de la région du (wadi) Masila'

\footnotetext{
32 házereh a pour sens premier 'espace laissé par une dent tombée' puis 'précipice' ( $c f$. házreh, LS 170).

${ }^{33}$ La transcription est celle de Morris, ce qui explique la différence entre e- ici et $\varepsilon$ chez Johnstone (JL), il peut aussi s'agir de deux variétés dialectales différentes.

${ }^{34}$ Le nom est un PL./COLL. qui désigne les 'gens d'origine africaine, descendants d'esclaves, des dépendants, les Noirs en général'.
} 
(53) Met bōli $\quad 1=$ rḥabēt

gens GEN.PL.ville

'Les gens de la ville'

(54) J
aféhl
Ź-elhóti
DEF.gens GEN.PL-DEF.vaches
'The cow-men'

(JL 22)

En H, le GEN. PL. est rarissime :

(55) Ḥ eśnéwwet đe-ḥeyđōten

DEF.articulations GEN.SG-DEF.oreilles

(HL 121)

'jaw-joints' (lit. articulations des oreilles $=$ articulations temporomandibulaires)

et pas uniquement dans les noms composés :

(56) Ḥ̣ beyār đe-ḥe-gēnbet (Str. 60,1)

chameaux GEN.SG-DEF-Janabah

'Some camels of the Janabah'

Les exemples ci-dessus, montrent que, dans cette construction, le défini peut ne marquer aucun des constituants (43), ou l'un des deux (56) ou les deux $(54,55)$, comme dans les constructions directes.

REMARQUE

Dans un parler $\mathrm{Hb}^{35}$, en contact avec un parler śheri, le même locuteur utilise la construction avec la marque $\mathrm{d}=\mathrm{et}$, dans le même énoncé, celle avec la marque $\varepsilon=$, dans un $\mathrm{SN}$ où il n'y a pas de relation de possession :
$\mathrm{Hb} \quad \mathrm{ftih}$
đə $=$ dekəkənēt
ouverture
GEN = boutique
'L'ouverture de la boutique'
ftih $\quad$ đə/ $\varepsilon=$ dəkkūn
ouverture $\quad$ GEN $=$ boutiques
'L'ouverture des boutiques'

En soqotri, la construction indirecte avec $\mathrm{d}=$ domine très largement, pour exprimer la possession ou l'appartenance. On la trouve :

avec des noms de parties du corps
(57) S śēfər / Rə́śfəror də= đéyn
$\mathrm{cil} / \mathrm{cils} \quad$ GEN.SG $=$ œil
'Le cil/les cils' (la présence ici du déterminant est redondante)

${ }^{35} \mathrm{Il}$ faut rappeler que le $\mathrm{Hb}$, du moins la variété parlée au Yémen, n'a pas d'article, 
La deuxième partie du SN reste invariable (GEN. SG=N.SG) N. En Ḥ (ḤL 118), la construction est similaire śêf đ-āyn $<$ cheveux/ GEN=œil> .

En Met (variété sans pharyngale sonore), le syntagme à l'état construit s'est lexicalisé, et s'est figé en un nom simple : SG śəfrīn ; PL. śfərīyin $<$ śfr=?cyn ( $c f$. ML (373) śəfəryēn). Il faut noter que, dans un corpus plus ancien, Jahn (1902, 240) relève une construction indirecte avec un SG śôfer da ayn 'le cil de l'œil' ('Wimper des Auges'), et un PL. śferiôn la ayénten 'les cils des yeux' (les deux constituants sont au PL.).

(58) $\mathrm{S}$

$\begin{array}{ll}\text { Pațbi } & \text { diy }=\bar{\jmath} z \\ \text { pis.DU. } & \text { GEN.SG }=\text { chèvre }\end{array}$

'Les deux trayons de la chèvre'

(59) S Riṭob di=lehé

pis.PL. $\quad$ GEN.SG $=$ vache

'Les pis de la vache'

On a la même construction, exprimant l'appartenance, l'attribution, ou la possession, avec pour $\mathrm{D}^{\text {ant }}$ un nom propre, un titre :

(60) $\mathrm{S}$ bédiyeh di=máhra

désert GEN.SG = Mahra

'Le désert du Mahra'

(61) $\mathrm{S}$ warār $\mathrm{di}=$ șáțhan

taxes $\quad$ GEN.SG $=$ sultan

'Les taxes du sultan' (taxes dues au sultan de facto et de jure)

(62) $\mathrm{S}$ megídoh di = șáțhan

généalogie GEN.SG = sultan

'La généalogie du sultan'

Comme l'illustrent (59) et (61), l'accord en nombre de la marque GEN. avec le D ${ }^{\text {é }}$ est loin d'être systématique.

(63) S Rāṣihəl $\quad 1=$ Éfœ

origines GEN.PL $=$ gens

'Les origines des gens'

La construction indirecte a tendance, en $\mathrm{S}$. aussi, à se figer avec une marque invariable, et ce, selon un processus dont on peut retrouver des traces à partir des exemples suivants :

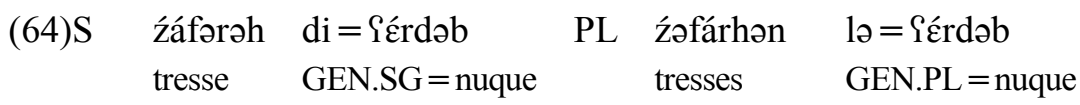

'La tresse de la nuque' et 'Les tresses de la nuque' 
Le syntagme s'est réduit à un lexème simple: au SG diférdəb, au PL lə^érdəb. Le GEN. ayant même forme que le démonstratif, le syntagme finit par signifier 'celle(s) de la nuque' ( $c f$. sous (37) iź-írún en JL). A partir de cet exemple, on peut comprendre qu'il y ait eu réinterprétation de la marque GEN, et recatégorisation du syntagme en un nom simple, à initiale $d i=a u ~ S G$, et $1=$ au PL. Un certain nombre de noms ayant pour $1^{\text {ère }}$ consonne radicale un d- ont eu un pluriel en 1-: dğeğ ${ }^{36}$ 'poule, poulet', PL. ilğeğ. Le lexème peut lui-même entrer dans une construction GEN marquée :

$\begin{array}{lll}\text { (65) } \mathrm{S} & \begin{array}{l}\text { maḥfeš } \check{s}^{37} \\ \text { local }\end{array} & \mathrm{d}=\text { ilğgeg } \\ & \text { GEN.SG }=\text { nuque } \\ & \text { poulailler' (Lit. local/endroit des poules) }\end{array}$

En B., la construction indirecte se distingue par une marque de GEN. /e/, à rapprocher $\mathrm{du} / \dot{\varepsilon} /$ en J. Comme en J, c'est à la fois la marque de DEF.et de GEN. La confusion entre DEF. et GEN. est impossible quand le $\mathrm{D}^{\text {ant }}$ est un nom propre (intrinsèquement défini)

(66) $\mathrm{B}$

$$
\begin{aligned}
& \text { məSaali erəkəbiit } \\
& \text { crique } \\
& \text { 'The inlet of Requebit' }
\end{aligned}
$$

(67) $\mathrm{B}$ ben $^{38} \quad$ emesmuun

(Morris 1983, 139)

fils GEN.Mesmun

(Morris 1983, 136)

'The son of Mesmuun'

Une autre construction en B. permet d'exprimer une relation d'appartenance. Le deuxième constituant du syntagme est précédé de $1(\partial)$. Il s'agit non pas du déterminant démonstratif (pl.) ni du REL. mais de la préposition qui désigne le destinataire, le bénéficiaire. La construction équivaut à $\mathrm{N}_{1}$ 'pour/à (for/to)' $\mathrm{N}_{2}{ }^{39}$ Cette construction n'est pas attestée avec cette valeur dans les autres langues.
$(68) \mathrm{B}$
ekáa
lə bətáh̆hriit
DEF.terre/pays GEN. Batahari(s)
(Morris 1983, 130)
'The land of Bațāhirah'
(69) B

śebh lo kəliit
graisse GEN. reins
'The fat of the kidneys'
(Morris 1983, 135)
'The fat of the kidneys'

\footnotetext{
${ }^{36}$ LS (120) atteste au SG degógoh (avec marque de f.) et au PL. Pílgog.

${ }^{37}$ Emprunt à l'arabe.

${ }^{38}$ Remarquons que ce nom de parenté a une forme différente quand il est dans une construction directe ( $c f$. (1), ber Yaliy).

${ }^{39} C f$., dans certains parlers français : 'C'est la voiture à son père', 'le fils à Jean'.
} 
En (68) seul le D ${ }^{e}$ est DEF., et en (69) aucun des constituants.

\section{2.2 Spécificité du soqotri : $\{G E N=P R . P E R S\}+N$}

Nous avons vu que rares sont les cas où le soqotri exprime la possession par un substitut pronominal post-clitique au nom. Seuls quelques noms de parenté sont concernés : 'père', 'mère', 'fils/fille', 'mari qui signifie aussi maître', 'maîtresse'. Quand le possesseur a un référent pronominal, la construction toujours directe dans les autres langues SAM, est indirecte en S.

Le pronom personnel, sous sa forme indépendante, entre dans une construction indirecte, $\mathrm{di}=$ lui est enclitique. Plus rarement, le pronom sous sa forme indépendante est post-clitique de mən, réduit parfois à $\mathrm{m}$ - (préposition marquant l'origine). Le syntagme ainsi formé est toujours préposé au nom $\mathrm{D}^{\mathrm{e}}$ seul, ou au nom $\mathrm{D}^{\mathrm{e}}$ précédé de la préposition qui marque sa fonction.

L'ordre des mots est toujours : $\{$ GEN=PR.PERS $\}+\mathrm{N}$,

$$
\text { et }\{\text { GEN }=\text { PR.PERS }\}+\text { (PREP.-)N. }
$$

Ces traits d'ordre morphologique et syntaxique permettent de distinguer le $\mathrm{S}$. à l'intérieur du SAM et le rapproche par sa syntaxe de certaines constructions dans des langues afro-sémitiques du nord : dahalik, tigré, tigrinya.
(70) $\mathrm{S} \quad \mathrm{di} / \mathrm{mon}=$ yheh šhem
GEN/PREP $=$ PR.PERS.3M.SG nom
'Son nom'

(71) $\mathrm{S}$
$\mathrm{m}=$ yeh
məḳáșaș
PREP $=$ PR.PERS.3M.SG moustache
'Sa moustache'

Devant un nom composé :

$$
\begin{array}{ll}
\mathrm{di}=\text { hœ } & \text { bər }=\text { ḳaḳa } \\
\text { GEN = PR.PERS.1.SG } & \text { fils = sœur/frère } \\
\text { 'Mon neveu' } &
\end{array}
$$

Devant un SN prépositionnel

$$
\begin{array}{ll}
\text { di }=\text { han } & \text { mən-half } \\
\text { GEN=PR.PERS.PL } & \text { hors_de-village } \\
\text { 'Hors de notre village' }
\end{array}
$$

$$
\begin{array}{lll}
\text { Pégah } & \mathrm{di}=\mathrm{hi} & \text { di-méglis } \\
\text { monter.ACC.3M.SG } & \mathrm{GEN}=\text { PR.PERS.3M.SG } & \text { vers-salon } \\
\text { 'Il monta vers/à sa pièce de repos' } &
\end{array}
$$

Deux exemples tranchent par leur syntaxe : l'un (75) provient d'un texte enregistré en 1997 auprès d'un locuteur de la côte nord, l'autre (76), d'un texte recueilli en décembre 2010 près de Ras Momi (est de l'île). Les textes plus anciens attestent d'un ordre immuable dans ces constructions. 

(75) $\mathrm{S}$ tahéroh de- $\mathrm{d}=$ seh bébəh
aller.ACC.FM.SG vers- GEN $=$ PR.PERS.3F.SG père
'Elle alla chez son père'

Le syntagme possessif est préposé au $\mathrm{D}^{\mathrm{e}}$, mais placé entre la PREP. et le N. qu'elle régit. L'ordre canonique serait : tahéroh dsé debébəh avec le déterminant possessif précédant tout le $\mathrm{SN}$ (prép. $+\mathrm{N}$ ).
(76) $\mathrm{S}$ zérieh $\mathrm{di}=$ ho
plantation GEN $=$ PR.PERS.1SG
'Mes plants'

Le possessif est placé après le $\mathrm{D}^{\mathrm{e}}$ (comme dans les autres langues SAM), au lieu de diho zérieh.

\section{L'annexion complexe}

Elle concerne les syntagmes où la relation de possession ou d'appartenance met en rapport un constituant nominal avec un autre nom, lui-même en état d'annexion avec un nom ou un substitut pronominal. Cette construction peut être récursive :

$\mathrm{A}$ appartient à $\mathrm{B}$ qui appartient à $\mathrm{C}$...(C pouvant être un $\mathrm{N}$ ou un $\mathrm{PR}$.).

Dans ce cas, comme dans celui d'une construction simple, l'état construit entre A et B n'est possible que lorsque A est un nom de parenté ou de partie du corps. La construction des autres constituants est indirecte et marquée comme dans l'annexion simple de deux éléments. L'ordre est toujours $\mathrm{D}^{\mathrm{e}}+\mathrm{D}^{\text {ant }}$, dans chaque segment, sauf en S. quand c'est un pronom personnel qui réfère au possesseur :

(77) Met hayb g̀a $\quad$ də=mbōrək
père frère
'Le père du frère de Mborek'

(78) Met hərūh đə=habr $=$ es (parler bédouin)

tête $\quad \mathrm{GEN}=$ fils $=$ PR.3F.SG

'La tête de son fils'

(79) Met tōdeğ tōdi đə $=$ hām $=$ is $\quad$ (parler bédouin)

3F.SG.INAC.téter sein.DU. GEN $=$ mère $=$ PR.3F.SG

'Elle tète les (deux) seins de sa mère'

(80) Met hēm habūn le=habení=həm

PR.PERS.3M.PL enfants GEN.PL $=$ enfants $=$ SUF.3M.PL

'Eux, (ce sont) les enfants de leurs enfants (leurs petits-enfants)'

(81) H tę đe-hébes

(Str. 44, 14)

femme GEN.SG-père.PR.3F.SG

'Her father's wife' 
teł đe-bāl aṣayd (Str. 42, 6)

femme GEN.SG-reponsable DEF.poisson

'The fisherman's wife'

(83) Met hağerīt $d=$ bōli-āmēn

dépendants GEN.SG=gens-SOman

'Les Noirs anciens dépendants des Omanais'

(82) et (83) montrent que le syntagme basé sur/baSal/ fonctionne bien comme un nom composé (pas de marque de GEN entre ses deux constituants)
$\varepsilon t+b$
É-ใÉmóš
DEF.téton GEN.SG-mère.PR.2M.SG
'The teat of his mother'

(JL )

En S. le possessif est préposé au nom marqué comme GEN.

(85)
bīyəh $\mathrm{di}=$ hœ
di $=$ bébeh
mère $\quad$ GEN=PR.PERS.1SG GEN=père
'La mère de mon père'

(86) $\mathrm{S}$
mon šn $\check{s}^{\mathrm{n}}$
$\mathrm{d}=$ ihi*
di $=$ bébeh
INT. GEN=PR.PERS.3M.SG
GEN=père

'Quel (est) le nom de son père?'

Tableaux récapitulatifs

1. Les marques de génitif (construction analytique)

\begin{tabular}{|c|c|c|c|c|c|c|}
\hline N.D $\mathrm{D}^{\mathrm{e}}+\mathrm{N} \cdot \mathrm{D}^{\mathrm{ant}}$ & $\mathrm{M}$ & $\mathrm{Hb}$ & $\mathrm{H}$ & $\mathrm{B}$ & $\mathrm{J}$ & $\mathrm{S}$ \\
\hline $\begin{array}{l}\mathrm{D}^{\hat{e}}+\mathrm{SG}=\mathrm{N} \cdot \mathrm{SG} / \mathrm{PL} \\
\mathrm{D}^{\hat{\varepsilon}}+\mathrm{PL}=\mathrm{N} \cdot \mathrm{SG} / \mathrm{PL}\end{array}$ & $\begin{array}{l}\mathrm{d} / \mathrm{d}= \\
\mathrm{l}=\end{array}$ & $\begin{array}{l}\mathrm{d}= \\
\mathrm{l}=\end{array}$ & $\begin{array}{l}\mathrm{d}= \\
\mathrm{l}=\end{array}$ & $\mathrm{e}=$ & $\begin{array}{l}\varepsilon= \\
\text { iź }=\end{array}$ & $\begin{array}{l}\mathrm{d}= \\
\mathrm{l}=\end{array}$ \\
\hline invariable & $\varnothing$ & $\varnothing$ & $\varnothing$ & $1-$ & $\varnothing$ & $\varnothing$ \\
\hline avec PR.PERS. & $\varnothing$ & $\varnothing$ & $\varnothing$ & $\varnothing$ & $\varnothing$ & $\mathrm{d}=\mathrm{PR} . /(\mathrm{m}($ (ən) $)=\mathrm{PR}$. \\
\hline
\end{tabular}

2. Ordre des constituants dans un syntagme génitival

\begin{tabular}{|l|l|l|l|l|l|l|}
\hline $\mathrm{D}^{\mathrm{e}}+\mathrm{D}^{\text {ant }}$ & $\mathrm{M}$ & $\mathrm{Hb}$ & $\mathrm{H}$ & $\mathrm{B}$ & $\mathrm{J}$ & $\mathrm{S}$ \\
\hline $\mathrm{N}+\mathrm{N}$ & + & + & + & $(+)^{*}$ & - & + \\
\hline $\mathrm{N}=$ PR.SUF & + & + & + & + & - & $(+)^{*}$ \\
\hline $\mathrm{N}+\mathrm{GEN}=\mathrm{N}$ & + & + & + & + & + & + \\
\hline $\mathrm{d}=$ PR PERS + (PREP) N. & - & - & - & - & - & + \\
\hline
\end{tabular}

$(+)^{*}$ occurrence très rare.

\section{Conclusion}

Dans toutes les langues SAM, l'ordre des mots dans une construction génitivale est toujours : déterminé + déterminant, quand les deux éléments de la relation sont 
des noms, en état construit ou non. La construction synthétique entre deux noms est beaucoup moins courante que la construction synthétique, elle est dépendante du champ sémantique auquel appartient le $\mathrm{D}^{\mathrm{e}}$; les deux noms doivent être dans une relation de possession dite inaliénable, d'appartenance inhérente. Excepté en soqotri, seule la construction directe est possible quand le possesseur a pour référent un pronom personnel. Il faut aussi remarquer que certains noms de parenté, de même radical lexical, ont une forme différente en construction directe et en construction indirecte, et ce dans toutes les langues. Ce point devrait faire l'objet d'une étude plus poussée.

La marque de génitif a la même base que le déterminant déictique ou le relateur. Elle est toujours clitique au N. $\mathrm{D}^{\text {ant }}$, deuxième constituant du syntagme. Invariable en genre, elle peut varier en nombre, en fonction de celui du premier constituant (le N. $\mathrm{D}^{\mathrm{e}}$ ), mais la construction a tendance à se figer au SG.

Le soqotri se caractérise par une construction originale lorsque c'est un pronom personnel qui réfère au possesseur. Le pronom, à la forme indépendante, est postclitique à la marque de génitif. Le syntagme ainsi formé précède le nom déterminé : l'ordre est alors inversé avec $\mathrm{D}^{\text {ant }}+\mathrm{D}^{\mathrm{e}}$. Ce trait rapproche le soqotri de certaines langues éthio/afro-sémitiques.

Dans les langues où est attesté un article défini, tout constituant nominal entrant dans une construction génitivale (directe ou indirecte) peut être défini par l'article. Ce trait spécifique au SAM est partagé par d'autres langues sémitiques méridionales parlées en Erythrée et en Ethiopie.

Enfin, il faut relever qu'un certain nombre de ces constructions génitivales se sont figées lexicalement. C'est surtout à partir du soqotri qu'on peut appréhender le sens du processus à l'œuvre, avec renouvellement de la morphologie nominale. La comparaison avec d'autres langues du sémitique méridional, à commencer par le dahalik répertorié récemment et parlé, comme le soqotri, exclusivement dans des îles (en Erythrée), devrait nous donner un nouvel éclairage sur le fonctionnement et la valeur de ces constructions et leur impact sur la morphosyntaxe en sémitique.

\section{Bibliographie}

Bittner, M. 1913, Studien zur Laut- und Formenlehre der Mehri-Sprache in Südarabien. III. Zum pronomen und zum Numerale $=$ Sitzungsberichte der Kais. Akademie des Wissenschaften in Wien. Philosophisch-Historische Klasse, 172/5, Wien: A. Hölder. Edzard, L. 2009, "Complex Annexations in Semitic", in: J.C. Watson - J. Retsö (eds), Relative Clauses and Genitive Constructions in Semitic $=$ Journal of Semitic Studies, Sup. 25, Oxford: Oxford University Press, pp. 51-64.

Bravmann, M.M., 1961, "Genetic aspects of the genitive in the Semitic languages", in Journal of the American oriental Society, vol. 81, n.4/Sept-Dec., pp. 386-394.

Creissels, D. 2006, Syntaxe Générale, une introduction typologique, vol.1, Paris: Hermès. 
Jahn, A. 1902, Die Mehri-Sprache in Südarabien. Texte und Wörterbuch = Kaiserliche Akademie der Wissenschaften. Südarabische Expedition, Bd III, Wien, Alfred Hölder.

Johnstone, T.M. 1977, Ḥarsūsi Lexicon and English- Ḥarsūsi Word-List, London: Oxford University Press.

Johnstone, T.M. 1981, Jibbāli Lexicon, London: Oxford University Press.

Johnstone, T.M. 1987, Mehri Lexicon and English-Mehri Word-List, with Index of the English Definitions in the Jibbāli Lexicon, compiled by G. Rex Smith, London: School of Oriental and African Studies.

Leslau, W. 1938, Lexique soqoți (sudarabique moderne) avec comparaisons et explications étymologiques, Paris: Klincksieck.

Morris, M. 1983 "Some Preliminary Remarks on a Collection of Poems and Songs of the Batahirah", in Journal of Oman Studies 6/1, pp. 129-144.

Morris, M. 1985 "A poem in Jibbaali", in Journal of Oman Studies 7, pp. 121130.

Müller, D.H. 1905, Die Mehri- und Soqoțri-Sprache. II. Soqoțri-Texte = Kaiserliche Akademie der Wissenschaften. Südarabische Expedition, Bd VI, Wien: Alfred Hölder.

Müller, D.H. 1907, Die Mehri- und Soqoți-Sprache. III Šhauri-Texte. Wien: Alfred Hölder.

Rodgers, J. 1991, "The Subgrouping of the South Semitic languages" in :Semitic Studies, 2, pp. 1323-1335.

Sima, A. 2009, Mehri-Texte aus der jemenitischen Šarqīyah, J.C. Watson - W. Arnold (eds) = Semitica Viva 47, Wiesbaden: Harrassowitz.

Simeone-Senelle, M.-Cl. 2003. " De quelques fonctions de $\underline{\mathrm{d}}-\mathrm{dans}$ les langues sudarabiques modernes", in : S. Robert (ed.), Perspectives synchroniques sur la grammaticalisation. Polysémie, transcatégorialité et échelles syntaxiques = Afrique et Langage 5, Louvain-Paris: Peeters, pp. 239-252.

Simeone-Senelle, M.-Cl. 2011, "Modern South Arabian", in S. Weninger (ed), The Semitic languages. An International Handbook, Berlin, New York: De Gruyter Mouton, pp. 1073-1113.

Stroomer, H. 1999, Mehri Texts from Oman. Based on the Field Materials of T.M. Johnstone $=$ Semitica Viva 22, Wiesbaden: Harrassowitz.

Stroomer, H. 2004, Ḥarsūsi Texts from Oman. Based on the Field Materials of T.M. Johnstone $=$ Semitica Viva 34, Wiesbaden: Harrassowitz.

Wagner, E. 1953, Syntax der Mehri-Sprache unter Berücksichtigung auch der anderen neusüdarabischen Sprachen $=$ Deutsche Akademie der Wissenschaften zu Berlin. Institut für Orientforschung 13, Berlin: Akademie-Verlag

Watson, J.C. 2009, "Annexion, Attribution and Genitives in Mahriyyōt", in: J.C. Watson - J. Retsö (eds), Relative Clauses and Genitive Constructions in Semitic = Journal of Semitic Studies, Sup. 25, Oxford: Oxford University Press, pp. 229244. 